\title{
COMPLETELY CONSERVATIVE DIFFERENCE SCHEMES FOR MAGNETOHYDRODYNAMIC EQUATIONS*
}

\author{
YU. P. POPOV and A. A. SAMARSKII \\ Moscow
}

(Received 17 December 1969)

1. IT was shown in [1] that the usual difference schemes, also including conservative oncs, have defects: they infringe the energy balance relations.

A class of schemes called completely conservative, which are free from this defect, exist. These schemes satisfy not only the difference analogs of the fundamental laws of the conservation of mass, momentum and total energy (as for ordinary conservative schemes), but the detailed energy balance is also valid, that is, the balance with respect to the individual forms of energy: internal and kinetic.

Completely conservative difference schemes may be obtained, for example, by means of a well-known integro-differential method [2] when a formal selection rule is observed. The essence of this rule is as follows. In gas dynamics the equation of the energy, for example, can be written in different forms: divergent, describing the variation in time of the total energy, non-divergent, expressing the variation of the internal energy, and entropic. In differential form these types are equivalent, that is, they reduce to one another by means of the other equations of the system.

But in the difference form this equivalence property does not in general hold, and is valid only for completely conservative schemes. In other words, completely conservative difference schemes simultaneously approximate the possible equivalent form of the original differential system of equations.

In this paper we consider difference schemes for magnetohydrodynamics in Lagrangian coordinates for the case of one spatial variable. Completely conservative difference schemes with the first and second order of approximation are constructed.

*Zh. vychisl. Mat. mat. Fiz., 10, 4, 990-998, 1970. 
2. A system of one-dimensional plane non-stationary equations of magnetohydrodynamics in the absence of the longitudinal component of the magnetic field in Lagrangian mass coordinates has the form [3]

$$
\begin{gathered}
\frac{\partial v}{\partial t}=-\frac{\partial p}{\partial x}+F, \\
\frac{\partial r}{\partial t}=v, \\
\frac{\partial}{\partial t}\left(\frac{1}{\rho}\right)=\frac{\partial v}{\partial x}, \\
\frac{\partial}{\partial t}\left(\frac{H}{\rho}\right)=\frac{\partial E}{\partial x}, \\
\frac{\partial H}{\partial x}=4 \pi \frac{I}{\rho}, \\
I=\sigma E, \\
\frac{\partial \varepsilon}{\partial t}=-p \frac{\partial v}{\partial x}+Q, \quad Q=\frac{I E}{\rho} .
\end{gathered}
$$

Notation: $t$ is the time, $r$ is an Eulerian variable, $\rho$ is the density of the medium, $x(d x=\rho d r)$ is a Lagrangian mass coordinate, $v$ is the longitudinal component of the velocity, $p$ is the gas pressure, $t$ is the internal energy, $\sigma$ is the electrical conductivity of the medium, $H, E$ are the transverse mutually perpendicular components, of the magnetic and electric fields, respectively, $I$ is the electric current density, $F$ is the Lorentz electromagnetic force, and $Q$ is the Joule heat. The time derivative is Lagrangian.

The system of equations of magnetohydrodynamics can be written in several equivalent forms possessing direct physical significance. Thus, the energy equation (2.7) taking equation (2.3) into account can also be represented in a non-divergent form, expressing the variation of the internal energy:

$$
\frac{\partial \varepsilon}{\partial t}=-p \frac{\partial}{\partial t}\left(\frac{1}{\rho}\right)+Q
$$

Moreover, equation (2.7), using (2.1) $-(2.5)$, can be reduced to the divergent form

$$
\frac{\partial}{\partial t}\left(\varepsilon+0.5 v^{2}+\frac{H^{2}}{8 \pi \rho}\right)=-\frac{\partial}{\partial x}\left[\left(p+\frac{H^{2}}{8 \pi}\right) v\right]+\frac{\partial}{\partial x}\left(\frac{E H}{4 \pi}\right)
$$

describing the variation of the total energy.

The differential equations $(2.7)-(2.9)$ are equivalent in the sense that they 
are reduced to one another by means of the remaining equations of the system.

In the equation of motion (2.1) the Lorentz force $F$ can be recorded in divergent and non-divergent forms, which are also equivalent:

$$
F=-\frac{I H}{0}=-\frac{\partial}{\partial x}\left(\frac{H^{2}}{8 \pi}\right) \text {. }
$$

In a numerical solution by the method of finite differences the system of differential equations is approximated by some difference scheme. The difference scheme can be constructed on the basis of any of the equivalent forms of the differential system. In particular, the construction of the usual conservative schemes is based on the divergent equations.

We formulate a difference scheme approximating the system of magnetohydrodynamic equations in the form $(2.1)-(2.7)$.

For this, in the part of the $x t$-space considered, we introduce the difference net $\left\{x_{i}, t^{j}\right\}, x_{i+1}=x_{i}+m_{i}, i=0,1, \ldots, N-1 ; t^{j+1}=t^{j}+\tau^{j}, j=0,1, \ldots$ For simplicity we will consider the net uniform $\left(m_{i}=m=\right.$ const, $\tau^{j}=\tau=$ const), although all the results obtained below are also valid for the case of non-uniform nets.

We define the net functions on the net, the values of the net functions of the velocity $v_{i}^{j}$, Eulerian variable $r_{i}{ }_{i}$, electric field intensity $E_{i}{ }^{j}$, current density $I^{j}$ and Lorentz factor force $F_{i}{ }_{i}^{j}$ will be referred to the nodes of the net $\left(x_{i}, t^{j}\right)$, and the values of the net functions of the density $\rho_{i}^{j}$, pressure $p_{i}{ }^{j}$, internal energy $\epsilon_{i}{ }^{j}$, magnetic field $H_{i}{ }^{j}$, electrical conductivity $\sigma_{i}{ }^{j}$ and Joule heat $Q_{i}{ }^{j}$ to the middles of the mass intervals $\left(x_{i+1 / 2}, t^{j}\right), x_{i+1 / 2}=x_{i}+0.5 m$.

Below, following [4], we will use the following index-free notation which will be convenient for calculations

$$
\begin{aligned}
& y_{i}^{j}=y, \quad y_{i}^{j+1}=\hat{y}, \quad y^{(0)}=\sigma \hat{y}(1-\sigma) y, \quad y( \pm 1)=y_{i \pm 1}^{j}, \\
& \frac{\hat{y}-y}{\tau}=y_{t}, \quad \frac{y(+1)-y}{m}=y_{x}, \quad \frac{y-y(-1)}{m}=y_{\bar{x}}
\end{aligned}
$$

$\sum_{i=1}^{N-1} y_{i} u_{i} m_{i}=(y, u), \quad \sum_{i=0}^{N-1} y_{i} u_{i} m_{i}=[y, u), \quad \sum_{i=0}^{N} y_{i} u_{i} m_{i}=(y, u]$.

For the summation of differences the following rule holds: 


$$
\left[y, u_{x}\right]=-\left[y_{\bar{x}}, u\right]+y_{N} u_{N}-y_{-1} u_{0}
$$

The formula

$$
(y \cdot u)_{t}=\hat{y} u_{t}+u y_{t}
$$

will be used in calculations for the differenoe differentiation of a product with respect to time, and the difference identity

$$
y^{\left(\sigma_{1}\right)}=y^{\left(\sigma_{2}\right)}+\left(\sigma_{1}-\sigma_{2}\right) \tau y_{t}
$$

will also be used.

3. We consider the following multiparameter family of difference schemes approximating the equations of magnetohydrodynamics:

$$
\begin{gathered}
v_{t}=-p_{\bar{x}}^{\left(\sigma_{1}\right)}+F, \\
r_{t}=v^{\left(\sigma_{2}\right)}, \\
\left(\frac{1}{\rho}\right)_{t}=v_{x}^{\left(\sigma_{3}\right)}, \\
\left(\frac{H}{\rho}\right)_{t}=E_{x}^{\left(\sigma_{4}\right)}, \\
H_{\bar{x}}=4 \pi \frac{I}{\rho(-1)}, \\
I=\sigma(-1) E, \\
\varepsilon_{t}=-p^{\left(\sigma_{5}\right)} v_{x}^{\left(\sigma_{\theta}\right)}+Q, \quad Q=\left(\frac{I}{\rho(-1)}\right)^{\left(\sigma_{7}\right)} E^{\left(\sigma_{8}\right)} .
\end{gathered}
$$

Here $0 \leqslant \sigma_{k} \leqslant 1, k=1,2, \ldots, 8$, are the parameters of the scheme, enabling the specific form of the time interpolation of the corresponding terms of the equations to be chosen.

In order to obtain a unified notation at the boundary points of the net for the equations of the scheme, and also for the difference formulas encountered below we introduce two fictitious net intervals $m_{-1}=0$ and $m_{N}=0$. Then, for example, in (3.1) we must understand by $p_{-1}$ and $p_{N}$ the values of the pressure net function at the boundary nodes, whereupon equation (3.1) itself still applies for $i=0$ and $i=N$.

Obviously, for the scheme (3.1)-(3.7) the law of conservation of mass is 
satisfied automatically because of the use of the mass coordinates. In this case, instead of it, it is necessary to require the satisfaction of some relation for the volume

$$
\sum_{i=0}^{N-1} \frac{1}{\rho_{i}} m_{i}=\left[\frac{1}{\rho}, 1\right)=r_{N}-r_{0}
$$

which by [1] is valid for

$$
\sigma_{3}=\sigma_{2}
$$

The use in the difference scheme of the non-divergent energy equation ensures the satisfaction of the internal energy balance

$$
\begin{gathered}
E^{j_{2}}-E^{j_{1}}=\tau \sum_{j=j_{1}}^{\boldsymbol{j}_{2}} A^{j}+B^{j} \\
E^{j}=[\varepsilon, 1), \quad A^{j}=-\left[p^{\left(\sigma_{s}\right)}, v_{x}^{\left(\sigma_{6}\right)}\right), \quad B^{j}=[Q, 1) .
\end{gathered}
$$

The relation (3.9) is obtained by summation over the net of equation (3.7) for $0 \leqslant i \leqslant N-1, j_{1} \leqslant j \leqslant j_{2}$ and expresses the fact that the variation of the internal energy of a fixed mass of gas $\left[x_{0}, x_{N}\right\rfloor$ occurs as a result of the total work of the pressure forces on the gas $A$ and the Joule heat $B$.

In the scheme (3.1)-(3.7) we calculate the variation of the total energy. Using (2.14) and (2.16) we transform the expression for $A$ :

$$
A^{j}=-\left[p^{\left(\sigma_{s}\right)}, v_{x}^{\left(\sigma_{6}\right)}\right]=\left[p_{x}^{\left(\sigma_{1}\right)}, v^{\left(\sigma_{6}\right)}\right]-R^{i}+D_{1}{ }^{j},
$$

where

$$
R^{j}=p_{N}^{\left(\sigma_{s}\right)} v_{N}^{\left(\sigma_{6}\right)}-p_{-1}^{\left(\sigma_{5}\right)} v_{0}^{\left(\sigma_{\theta}\right)}, \quad D_{1}^{i}=\left(\sigma_{5}-\sigma_{1}\right) \tau\left[p_{\overline{x t}}, v^{\left(\sigma_{s}\right)}\right] .
$$

After multiplying equation (3.1) by $v^{\left(\sigma_{6}\right)}$, summing it over the net and taking account of $(2.16)$ we obtain

$$
\begin{aligned}
{\left[p_{x}^{\left(\sigma_{1}\right)}, v^{\left(\sigma_{\theta}\right)}\right]-\left[F, v^{\left(\sigma_{0}\right)}\right] } & =-\left[v^{\left(\sigma_{6}\right)}, v_{t}\right]=-0.5\left[\left(v^{2}\right)_{l}, 1\right]+D_{2}{ }^{j} \\
D_{2}^{j} & =\left(0.5-\sigma_{6}\right) \tau\left[v_{t}{ }^{2}, 1\right]
\end{aligned}
$$

We also transform the electromagnetic terms:

$$
B^{j}=\left[\left(\frac{I}{\rho(-1)}\right)^{\left(\sigma_{\imath}\right)}, E^{\left(\sigma_{\mathrm{s}}\right)}\right)=\frac{1}{4 \pi}\left[H_{\frac{\left(\sigma_{\eta}\right)}{x}}, E^{\left(\sigma_{\mathrm{s}}\right)}\right)=
$$




$$
\begin{gathered}
=-\frac{1}{4 \pi}\left[H^{\left(\sigma_{7}\right)}, E_{x}^{\left(\sigma_{3}\right)}\right)+\Pi^{j}+D_{3}^{j} \\
\Pi^{j}=\frac{1}{4 \pi}\left(H_{N-1}^{\left(\sigma_{3}\right)} E_{N}^{\left(\sigma_{3}\right)}-H_{-1}^{\left(\sigma_{1}\right)} E_{0}^{\left(\sigma_{s}\right)}\right), \quad D_{3}^{j}=\frac{1}{4 \pi}\left(\sigma_{4}-\sigma_{8}\right) \tau\left[H^{\left(\sigma_{1}\right)}, E_{\overline{x t}}\right) .
\end{gathered}
$$

Here we use the difference equations for the electromagnetic field (3.4)-(3.5).

Starting from formula (2.15) for the difference differentiation of a product, it is possible to verify the validity of the difference identity

$$
\left(\frac{H^{2}}{\rho}\right)_{t}=2 H^{(0.5)}\left(\frac{H}{\rho}\right)_{t}-H \hat{H}\left(\frac{1}{\rho}\right)_{t},
$$

which after replacing the time derivatives in accordance with (3.3) and (3.4) can be rewritten in the form

$$
H^{(0.5)} E_{x}^{\left(\sigma_{3}\right)}=0.5\left(\frac{H^{2}}{\rho}\right)+0.5 H \hat{H} v_{x}^{\left(\sigma_{3}\right)}
$$

Taking into account the last relation, we continue the chain of transformations (3.12):

$$
\begin{gathered}
\frac{1}{4 \pi}\left[H^{\left(\sigma_{t}\right)} E_{x}^{\left(\sigma_{t}\right)}\right)=\left[\left(\frac{H^{2}}{8 \pi \rho}\right)_{t}, 1\right)+\left[\frac{H \hat{H}}{8 \pi}, v_{x}^{\left(\sigma_{3}\right)}\right)-D_{4}{ }^{j}= \\
{\left[\left(\frac{H^{2}}{8 \pi \rho}\right)_{i}, 1\right)-\left[\left(\frac{H \hat{H}}{8 \pi}\right)_{x}, v^{\left(\sigma_{t}\right)}\right]+\frac{H_{N} \hat{H}_{N}}{8 \pi} v_{N}^{\left(\sigma_{a}\right)}-} \\
\frac{H_{-1} \hat{H}_{-1}}{8 \pi} v_{0}^{\left(\sigma_{6}\right)}-D_{4}^{j}-D_{b}{ }^{j}, \\
\nu_{4}^{j}=\frac{1}{4 \pi}\left(0.5-\sigma_{7}\right) \tau\left[H_{t},\left(\frac{H}{\rho}\right)_{t}\right), \quad D_{5}{ }^{j}=\frac{1}{8 \pi}\left(\sigma_{6}-\sigma_{3}\right) \tau\left[H \hat{H}, v_{x t}\right) .
\end{gathered}
$$

Bringing together all the results in (3.9), we obtain the difference analog of the law of conservation of the total energy for a fixed mass $\left[x_{0}, x_{N}\right]$ in the time interval $\left[t^{\dot{j}_{1}}, t^{\hat{j}_{2}}\right]$ :

$$
\begin{gathered}
{\left.[\varepsilon, 1)\right|_{j_{1}} ^{j_{2}}+0.5\left[v^{2}, 1\right]\left|j_{j_{1}}^{j_{2}}+\left[\frac{H^{2}}{8 \pi \rho}, 1\right)\right|_{j_{1}}^{j_{2}}+\tau \sum_{j=j_{1}}^{j_{1}}\left\{\left(p_{N}^{\left(\sigma_{3}\right)}+\frac{H_{N} \hat{H}_{N}}{8 \pi}\right) v_{N}^{\left(\sigma_{0}\right)}-\right.} \\
\left.\left(p_{-1}^{\left(\sigma_{0}\right)}+\frac{H_{-1} \hat{H}_{-1}}{8 \pi}\right) v_{0}^{\left(\sigma_{0}\right)}-\Pi^{j}\right\}=\Delta E
\end{gathered}
$$




$$
\Delta E=\tau \sum_{j=j_{t}}^{j_{2}} \sum_{p=1}^{6} D_{p}{ }^{j}, \quad D_{\mathrm{e}}{ }^{j}=\left[\left(F+\frac{H \hat{H}}{8 \pi}\right)_{\bar{x}}, v^{\left(\sigma_{s}\right)}\right],
$$

It is obvious that this law is not satisfied in the general case. The unbalance of the total energy $\Delta E$ accumulates with time and for smooth solutions is of order $O(r)$. But for discontinuous and strongly varying solutions, as follows from the structure of the terms $D_{p}{ }^{j}$, the unbalance may become significant and considerably distort the nature of the solution.

It is noteworthy that the unbalance is independent of $m$ the step of the net, and hence cannot be decreased by thickening the spatial net.

The presence of unbalance of the total energy in the scheme considered is connected with the non-divergence of the energy equation (3.7). However, the use in the scheme of the divergent equation, for example, in the form

$$
\begin{aligned}
\left(\varepsilon+0.5 v^{2}+\frac{H^{2}}{8 \pi \rho}\right)_{t}=- & {\left[\left(p^{\left(\sigma_{5}\right)}(-1)+\frac{H(-1) \hat{H}(-1)}{8 \pi}\right) v^{\left(\sigma_{8}\right)}\right]_{x}+} \\
& \left(\frac{H^{\left(\sigma_{\gamma}\right)}(-1) E^{\left(\sigma_{8}\right)}}{4 \pi}\right)_{x}
\end{aligned}
$$

leads to similar difficulties. When the law of conservation of the total energy is satisfied in such a scheme, the detailed energy balance, that is, the balance for the separate forms of energy, will be violated. Thus, the balance for the internal energy has the form

$$
\begin{aligned}
& {\left.[\varepsilon, 1)\right|_{j_{1}} ^{j_{2}}-\tau \sum_{j=j_{1}}^{j_{2}}\left\{\left[p^{\left(\sigma_{1}\right)}, v_{x}^{(0.5)}\right)+\left[\left(\frac{I}{\rho(-1)}\right)^{(0.5)} E^{\left(\sigma_{4}\right)}\right)\right\}=\overline{\Delta E}} \\
& \overline{\Delta E}=\tau \sum_{j=j_{1}}^{j_{2}} \sum_{p=1}^{6} \bar{D}_{p^{j}} \\
& \bar{D}_{1}^{j}=\left(0.5-\sigma_{9}\right) \tau\left\{\left(p_{N-1}^{\left(\sigma_{1}\right)}+\frac{H_{N-1} \hat{H}_{N-1}}{8 \pi}\right)\left(v_{N}\right)_{t}-\left(p_{-1}^{\left(\sigma_{1}\right)}+\frac{H_{-1} \hat{H}_{-1}}{8 \pi}-\right)\left(v_{0}\right) t\right\} \\
& \bar{D}_{2}^{i}=\left(\sigma_{1}-\sigma_{5}\right) \tau\left\{\left(p_{X-1}\right)_{l} v_{N}^{\left(\sigma_{5}\right)}-\left(p_{-1}\right)_{1} v^{\left(\sigma_{\theta}\right)}\right\}, \\
& \bar{D}_{3}{ }^{j}=\left(\sigma_{7}-0.5\right) \tau\left\{\left(H_{N-1}\right)_{l} E_{N}^{\left(\sigma_{a}\right)}-\left(H_{-1}\right)_{l} E_{0}^{\left(\sigma_{s}\right)}\right\} \text {, } \\
& \bar{D}_{4}{ }^{j}=\left(\sigma_{3}-\sigma_{4}\right) \tau\left\{H_{N-1}^{(0,5)}\left(E_{N}\right)_{t}-H_{-1}^{(0.5)}\left(E_{0}\right)_{t}\right\} \text {, }
\end{aligned}
$$




$$
\vec{D}_{5}{ }^{j}=\left(\sigma_{3}-0.5\right) \tau\left[\frac{H \hat{H}}{8 \pi}, v_{x t}\right), \quad \bar{D}_{6}{ }^{j}=-\left[F+\left(\frac{H \hat{H}}{8 \pi}\right)_{\bar{x}}, v^{(0.5)}\right]
$$

In the general case $\overrightarrow{\Delta E} \neq 0$, which testifies to the bad approximation in the scheme for the internal energy. This defect of the scheme is no less dangerous than the violation of the law of conservation of the total energy. For example, in a number of calculations this has caused the appearance of such a non-physical effect as a decrease in the temperature of a certain mass of gas in the compression stage in the presence of Joule heating. Such phenomena are particularly inadmissible in the calculation of problems involving functions strongly dependent on the temperature, like the electrical conductivity, thermal conductivity, etc.

In order to ensure, in the scheme (3.1)-(3.7) the observance of the difference law of conservation of the total energy $\Delta E \equiv 0, D_{p}{ }^{j} \equiv 0$ in (3.13), and in the scheme with the divergent equation (3.14) the observance of the balance of the internal energy $\overline{\Delta E} \equiv 0, \bar{D}_{p}^{j} \equiv 0 \quad$ in (3.15), it is sufficient to satisfy the conditions

$$
\sigma_{5}=\sigma_{1}, \quad \sigma_{6}=\sigma_{3}=0.5, \quad \sigma_{7}=0.5, \quad \sigma_{8}=\sigma_{4}, \quad F=-\left(\frac{H \hat{H}}{8 \pi}\right)_{\bar{x}} .
$$

Here both schemes are equivalent, equation (3.7) reduces algebraically to the divergent equation (3.14) and conversely.

It is also obvious that the equation $\sigma_{6}=\sigma_{3}$ ensures the algebraic equivalence of equation (3.7) to the difference equation

$$
\varepsilon_{t}=-p^{\left(\sigma_{5}\right)}\left(\frac{1}{\rho}\right)_{t}+Q
$$

which approximates the differential equation (2.8).

The conditions (3.16) uniquely define the difference form of the Lorentz force $F$. This expression approximates the divergent differential form in (2.10) and ensures the observance in the scheme of the difference analog of the law of conservation of momentum.

The divergent difference expression for the force (3.16) can be transformed to the non-divergent form

$$
F=-0.25\left[\left(\frac{\hat{I}}{\rho(-1)}\right)(H+H(-1))+\frac{I}{\rho(-1)}(\hat{H}+\hat{H}(-1))\right],
$$


which approximates the non-divergent relation in $(2.10)$.

Therefore, the conditions (3.8) and (3.16) select from the eight-parameter family of difference schemes (3.1)-(3.7), approximating the system of equations of magnetohydrodynamics, a two-parameter family of schemes (with the free parameters $a$ and $\beta$ ), which are completely conservative:

$$
\begin{gathered}
v_{l}=-p_{\bar{x}}^{(\alpha)}-\left(\frac{H \hat{H}}{8 \pi}\right)_{\bar{x}}, \quad r_{l}=v^{(0.5)}, \quad\left(\frac{1}{\rho}\right)_{t}=v_{x}^{(0.5)}, \\
\left(\frac{H}{\rho}\right)_{l}=E_{x}^{(8)}, \quad H_{\bar{x}}=4 \pi \frac{I}{\rho(-1)}, \quad I=\sigma^{(-1)} E, \\
\varepsilon_{t}=-p^{(\alpha)} v_{x}^{(0.5)}+\left(\frac{I}{\rho(-1)}\right)^{(0.5)} E^{(\beta)} .
\end{gathered}
$$

For the family of schemes (I) not only the fundamental difference laws of the conservation of mass (volume), momentum and total energy are valid, but also a number of additional balance relations, the necessity for the satisfaction of which is dictated by physical considerations, for example, the detailed balance of energy, that is, balance with respect to the separate forms of energy - internal, kinetic, magnetic. The scheme (I) simultaneously approximates the possible equivalent forms of the system of differential equations of magnetohydrodynamics. The scheme has the order of approximation $O(r+m)$; for the parameter values $\alpha=\beta=0.5$ the order of approximation equals $O\left(\tau^{2}+m\right)$.

4. A family of completely conservative schemes, similar to the family (I), can be constructed by using a somewhat different difference approximation of the Joule heat in the energy equation (3.7):

$$
Q=\left(\frac{I(+1)}{\rho},\right)^{\sigma_{7}} E^{\left(\sigma_{8}\right)}(+1)
$$

Here the conditions of complete conservation (3.16) are preserved, and the energy equation (3.7) is equivalent to the following divergent equation:

$$
\left(\varepsilon+0.5 v^{2}(+1)+\frac{H^{2}}{8 \pi \rho}\right)_{t}=-\left[\left(p^{(\alpha)}+\frac{H H}{8 \pi}\right) v^{(0.5)}\right]_{x}+\left(\frac{H^{(0.5)} E^{(\beta)}}{4 \pi}\right)_{x} .
$$

Here another form of the approximation of the kinetic energy is used.

This family of schemes also has the order of approximation $O(\tau+m)$, and for $\alpha=\beta=0.5$ the order is $O\left(r^{2}+m\right)$.

The schemes constructed are recorded on unsymmetric patterns and hence 
have the first order of approximation with respect to space. By using a symmetric pattern it is possible to obtain completely conservative schemes with the second order of approximation:

$$
\begin{gathered}
v_{t}=-p_{\bar{x}}^{(\alpha)}-\left(\frac{H \hat{H}}{8 \pi}\right)_{\bar{x}}, \quad r_{t}=v^{(0.5)}, \quad\left(\frac{1}{\rho}\right)_{t}=v_{x}^{(0.5)}, \\
\left(\frac{H}{\rho}\right)_{t}=E_{x}^{(i)}, \quad H_{\bar{x}}=4 \pi \frac{I}{\rho_{*}}, \quad I=\sigma_{*} l, \\
\varepsilon_{t}=-p^{(\alpha)} v_{x}^{(0.5)}+0.5\left[\left(\frac{I}{\rho_{*}}\right)^{(0.5)} E^{(\beta)}+\left(\frac{I(+1)}{\rho_{*}(+1)}\right)^{(0.5)} E^{(\beta)}(+1)\right] .
\end{gathered}
$$

Here the notation $y *=0.5(y+y(-1))$ has been used.

The non-divergent energy equation in the scheme (11) is transformed algebraically to the following divergent form:

$$
\begin{aligned}
& \left(\varepsilon+0.25\left(v^{2}+v^{2}(+1)\right)+\frac{H^{2}}{8 \pi \rho}\right)_{t}= \\
& -\left[\left(p^{(\alpha)}+\frac{(H \hat{H}) \bullet}{8 \pi}\right) v^{(0.5)}\right]_{x}+\left(\frac{H^{(0.5)} E^{(\beta)}}{4 \pi}\right)_{x} .
\end{aligned}
$$

The value of the parameters $\alpha=\beta=0.5$ select from the family (II) the only completely conservative difference scheme for the equations of magnetohydrodynamics with the second order of approximation $O\left(r^{2}+m^{2}\right)$.

5. Completely conservative difference schemes approximating the system of equations of magnetohydrodynamics were realized in the calculation of a heavy current discharge in a plasma [5]. The processes generated in the plasma as the result of the discharge through it of a battery of capacitors were considered in the problem. As was shown by the calculations, a complex magnetohydrodynamic flow with large spatial gradients and sharp time variations of the parameters is produced.

When this problem was computed by the usual implicit schemes with a nondivergent energy equation [6] an unbalance of the total energy was observed, which in different versions amounted to $20-50 \%$ of the total energy of the system. This led to a physically absurd result: the energy emitted from the system in the form of optical radiation up to the end of the process 'exceeded the initial store of energy contained in the battery of capacitors.

The application of completely conservative schemes removed this defect.

6. Iteration methods are usually used for solving a system of implicit equations, 
the iteration process being continued until a given accuracy or a given number of iterations is reached. In practice this means that instead of the assumed difference scheme some other scheme is realized, depending on the method of solving the difference equations. Generally speaking, this scheme does not possess the property of complete conservation and is even not conservative. Hence in real calculations the unbalances will already not be accurately equal to zero. Their value depends on the number of iterations performed and may be used as an indication of the "iterability" of the scheme.

Because in completely conservative difference schemes the corresponding divergent and non-divergent difference equations are algebraically equivalent, it is a matter of indifference from the point of view of the satisfaction of the balance relations which particular form of the equation is used in the scheme. However, in practice it is more convenient to use the less laborious non-divergent equations.

Translated by J. Berry

\section{REFERENCES}

1. POPOV, YU. P. and SAMARSKII, A. A. Completely conservative difference schemes. Zh. vychisl. Mat. mat. Fiz., 9, 4, 953-958, 1969.

2. TIKHONOV, A. N. and SAMARSKII, A. A. Homogeneous difference schemes. $Z h$. vychisl. Mat. mat. Fiz., 1, 1, 5-63, 1961.

3. LANDAU, L. D. and LIFSHITS, E. M. Electrodynamics of Continuous Media (Elektrodinamika sploshnykh sred) Gostekhizdat, Moscow, 1957.

4. SAMARSKII, A. A. A priori estimates for the solution of the difference analogue of

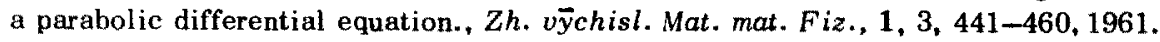

5. VOLOSEVICH, P. P., GOL'DIN, V. YA., KALITKIN, N. N., KURDYUMOV, S. P., POPOV, YU. P., ROZANOV, B. V., SAMARSKII, A. A. and CHETVERUSHKIN, B. N. Numerical Calculation of a Heavy Current Discharge in a Lithium Plasma. Ninth international conference on phenomena in ionised gases. Bucharest, 1969.

6. SAMARSKII, A. A., VOLOSEVICH, P. P., VOLCHINSKAYA, M. I. and KUDRYUMOV, S. P. A finite difference method for the solution of one-dimensional non-stationary problems of magnetohydrodynamics. Zh. vȳchisl. Mat. mat. Fiz., 8, 5, 1025-1038, 1968 . 